\title{
Experimental verification of the characteristic behaviors in passive dynamic walking
}

\author{
Masatsugu Iribe $^{1} \cdot$ Ryoichi Hirouji ${ }^{1}$. Daisuke Ura ${ }^{2} \cdot$ Koichi Osuka $^{2} \cdot$ Tetsuya Kinugasa $^{3}$
}

Received: 14 April 2020 / Accepted: 25 November 2020 / Published online: 4 January 2021

(C) The Author(s) 2021

\begin{abstract}
It is well known that passive dynamic walking shows chaotic behavior owing to changes in the environment. In addition, when the environment changes continuously during walking, passive dynamic walking shows "adaptive behaviors" in which the stride angle changes itself in an attempt to keep walking. These behaviors are very interesting and useful for the legged robot design. However, the studies on passive dynamic walking are preceded only by numerical simulations. For this reason, it is very important to confirm, by actual experiments, whether these characteristic behaviors appear. In this paper, we verify the existence of these behaviors by several actual experiments.
\end{abstract}

Keywords Passive dynamic walking $\cdot$ Adaptive behavior $\cdot$ Experimental verification

\section{Introduction}

It is well known that passive dynamic walking shows characteristic behaviors owing to changes in the internal or external environment [1-7]. In addition, passive dynamic walking shows "adaptive behaviors" in which a passive dynamic walking robot adjusts its gait to keep walking in a continuously changing walking environment during walking.

We showed how to design a legged robot capable of passive dynamic walking by applying those behaviors [8-10]. This makes it possible to determine clearly the size, mass, and joint number, which shows the degrees of freedom of a legged robot, of the passive dynamic walking robot by applying our proposed design method. The behaviors are very interesting and can show the feasibility of the legged

This work was presented in part at the 25th International Symposium on Artificial Life and Robotics (Beppu, Oita, January 22-24, 2020).

Masatsugu Iribe

iribe@osakac.ac.jp

1 Graduate School of Engineering, Osaka Electro-

Communication University, 18-8 Hatsu-cho,

Neyagwa 572-8530, Osaka, Japan

2 Graduate School of Engineering, Osaka University, 2-1 Yamadaoka, Suita 565-0871, Osaka, Japan

3 Faculty of Engineering, Okayama University of Science, 1-1 Ridai-cho, Kita-ku, Okayama 700-0005, Okayama, Japan robot design. However, the studies on passive dynamic walking are preceded by numerical simulations and have not been sufficiently verified by actual experiments.

For this reason, it is very important to confirm whether the above-mentioned characteristic behaviors actually appear. In this paper, we attempt to verify the existence of those characteristic behaviors, which are confirmed in numerical simulations of conventional studies, by several experiments.

\section{Characteristic behaviors of passive dynamic walking}

Passive dynamic walking is one of the walking motions, where gravity and the dynamics of the robot itself are used to walk down a gentle slope without any actuators or controls. During this walking motion, the stride angle, which is the absolute value of the difference between the forward leg angle and the backward leg angle at the two-leg supporting period, increases as the walking slope angle increases. In addition, when the walking slope angle exceeds a certain value, the stride angle begins to bifurcate. This bifurcation is called the chaotic behavior of a nonlinear system [2-4]. As described above, the stride angle and walking period change in response to changes in the environment, such as the change in the walking slope angle. 
Passive dynamic walking also shows characteristic behaviors. When changes in the environment occur continuously, a passive dynamic walking robot attempts to keep walking by adjusting its stride angle and walking period automatically in response to the changes. This behavior is called "adaptive behavior" $[10,11]$.

Adaptive behavior appears with not only environmental changes but also changes in internal parameters, such as the size, mass, and degrees of freedom of a legged robot. Thus, a legged robot capable of passive dynamic walking can be designed by applying adaptive behavior [10, 11]. In addition, it is possible to stabilize the walking motion by adjusting the mechanical impedance of joints, which are internal parameters of the legged robot $[8,9]$.

On the other hand, most of the research studies on adaptive behavior have been preceded by numerical simulations, and there are few verifications by experiments with an actually manufactured legged robot [4]. Thus, it is necessary to perform actual experiments using manufactured legged robots to clarify the existence of the adaptive function.

\section{Experimental environments}

\subsection{Legged robot capable of passive dynamic walking}

Figure 1 shows the legged robot that we developed, which is capable of passive dynamic walking. The legged robot has a near-complete symmetrical structure with respect to the sagittal plane, so it walks with a constant stride angle at each step. The robot can expand and contract the foot part about $10 \mathrm{~mm}$ by using the sensor information from the sole to avoid the collision of the foot of the swinging leg with the walking road surface. These motions are controlled by Arduino microcomputer. Principles of the leg extension

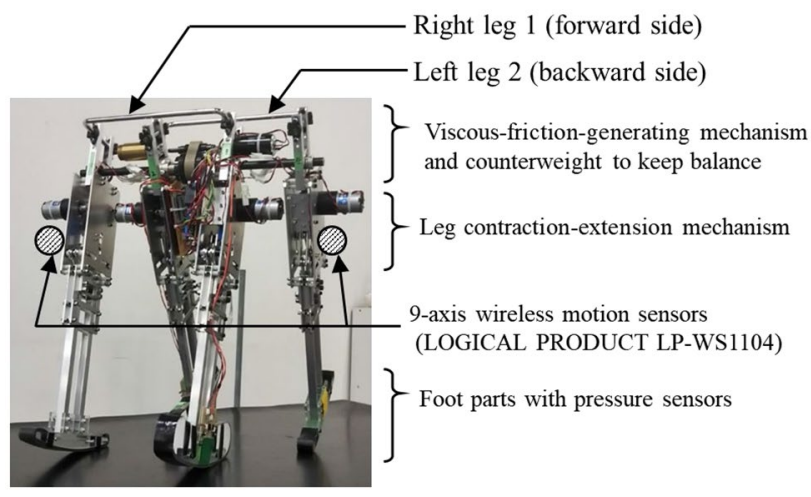

Fig. 1 Legged robot capable of passive dynamic walking through variable viscous-friction-generating mechanism and contraction mechanism and its motion control were explained in detail in a previous report [12].

The particular feature of the robot is the viscous-friction-generating mechanism. This mechanism controls the viscosity friction coefficient of the hip joint by applying the regeneration brake of a DC motor. Figure 2 shows an overview of the mechanism. The microcomputer as mentioned above controls the timing of terminal short of the DC motor and changes the viscosity friction coefficient dynamically.

The stride angle of the robot is measured using a small 9-axis wireless motion sensor module (LOGICAL PRODUCT LP-WS1104), which is attached to both legs. Built-in acceleration sensors and gyro sensors in the motion sensor module are used to measure the leg angle during walking. The initial angle of the leg is obtained by measuring the gravitational acceleration acting on the leg and calculating the arc sine function of the value. The acceleration sensors can measure gravitational acceleration with an accuracy of $1.53 \mathrm{E}-4 \mathrm{G} / \mathrm{bit}$. The leg angle during walking is obtained by calculating the numerically integrated value of the angular velocity of the leg, which is measured by the gyro sensors. These sensors can measure angular velocity with an accuracy of $0.0458(\% / \mathrm{s}) / \mathrm{bit}$. Each signal is acquired at a sampling frequency of $1 \mathrm{kHz}$, and the noise is removed using a low-pass filter. Since the walking motion is performed in about 0.7 to $0.8 \mathrm{~s}$ per step, the cut-off frequency of the low-pass filter is set to $10 \mathrm{~Hz}$.

Finally, we show the dynamical model of our developed legged robot in Fig. 3 and the specifications in Table 1 in detail.

\subsection{Walking slope with variable angle function}

Figure 4 shows the walking slope used in the experiments. This walking slope consists of four 2.0-m-long plates

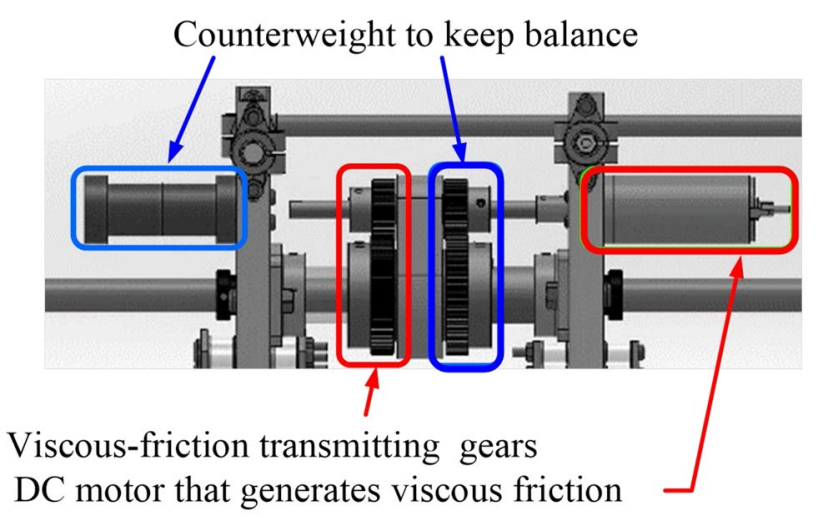

Fig. 2 Viscous-friction-generating mechanism 


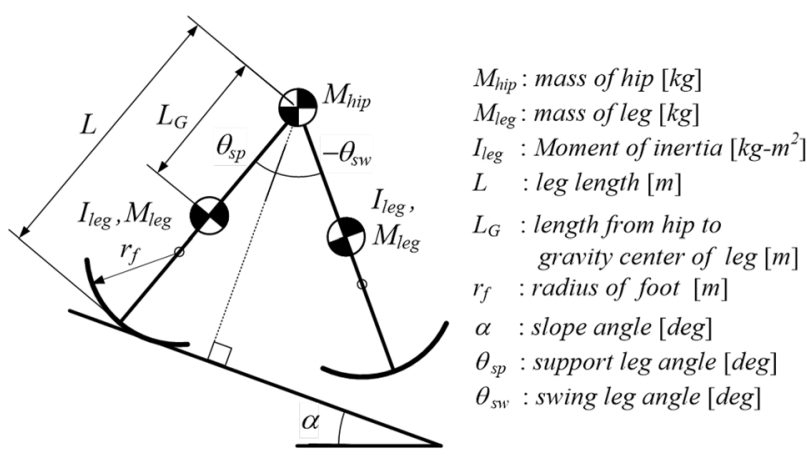

Fig. 3 System model of passive dynamic walking robot

connected together, so the length is $8.0 \mathrm{~m}$ in total. The inclination angle of the slope can be set for each plate in the range of $2.0^{\circ}-3.0^{\circ}$. The experiments were performed at inclination angles of $2.0^{\circ}, 2.5^{\circ}, 2.7^{\circ}$, and $3.0^{\circ}$.

\section{Verification by experiments}

\subsection{Relationship between slope angle and stride angle}

Figure 5 shows measurement results, that is, the leg angle trajectories of $\theta_{\mathrm{sp}}$ and $\theta_{\mathrm{sw}}$ of the left and right legs, when the inclination angles of the walking slope are $2.0^{\circ}$ and $2.5^{\circ}$. Typical trajectories of the leg angles in the time series and the configuration space diagram under each condition are shown in Fig. 5.

From these results, it can be confirmed that the stride angle increases with increasing walking slope angle. In particular, when the inclination angle of the walking slope is $2.5^{\circ}$, the variation of the stride angle becomes large, and the walking motion shows unstable behaviors. As the walking slope angle increases, the robot begins to show oscillation and unstable motion.

Next, we investigated how the stride angle changed when the walking slope angle was gradually increased. We investigated the stride angle changes when the inclination angle of the walking slope was increased in the order of $2.0^{\circ}$, $2.5^{\circ}, 2.7^{\circ}$, and $3.0^{\circ}$. The robot walked a distance of $8.0 \mathrm{~m}$ in 18 steps or more on the walking slope whose inclination angle was set to $2.0^{\circ}$ and $2.5^{\circ}$. The first 2 or 3 steps, which corresponded to the pull-in motion at the start of walking and the last step that disturbed the end of walking, were excluded. Then we collected walking data of 15 steps under the walking slope condition. On the other hand, because the stride angle increased as the walking slope angle were increased, the robot finished walking in 15 steps on the walking slope whose inclination angle was set to $2.7^{\circ}$ and $3.0^{\circ}$. Thus, we collected walking data of 12 steps on the walking slope whose inclination angle was set to $2.7^{\circ}$ and $3.0^{\circ}$. In addition, 10 walking experiments were performed under the same experimental conditions to further process the data statistically. The viscosity friction coefficient of the hip joint

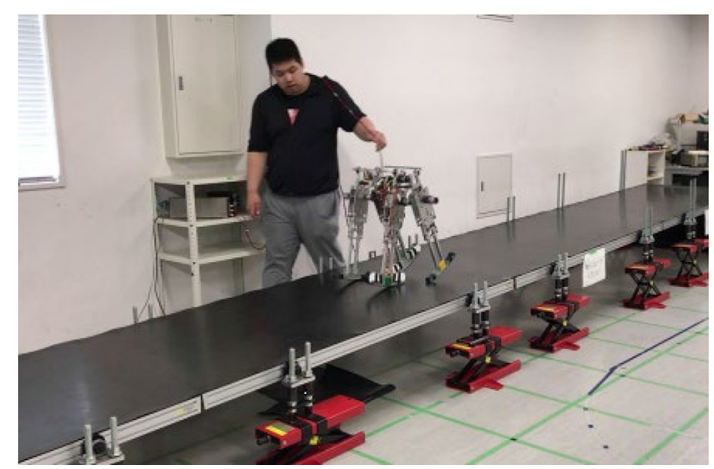

Fig. 4 Experiments on walking slope with adjustable inclination angle

Table 1 Specifications of legged robot

\section{Mass (kg)}

Height (mm)

Width (mm)

Mass of hip (kg)

Leg length (Length from hip to foot) (mm)

Foot length (mm)

Radius of foot curvature (mm)

\begin{tabular}{lll}
\hline & Right leg & Left leg \\
\hline Mass of leg $(\mathrm{kg})$ & 4.82 & 4.90 \\
Length from the hip to gravity center of leg $(\mathrm{mm})$ & 111.0 & 111.8 \\
Moment of inertia $\left(\mathrm{kg}-\mathrm{m}^{2}\right)$ & 0.419 & 0.419 \\
\hline
\end{tabular}

\begin{tabular}{|c|}
\hline 10.80 \\
\hline 630 \\
\hline 610 \\
\hline 1.08 \\
\hline $\begin{array}{l}540(530 \\
\text { when con- } \\
\text { tracted })\end{array}$ \\
\hline 300 \\
\hline 540 \\
\hline Left leg \\
\hline 4.90 \\
\hline 111.8 \\
\hline 0.419 \\
\hline
\end{tabular}



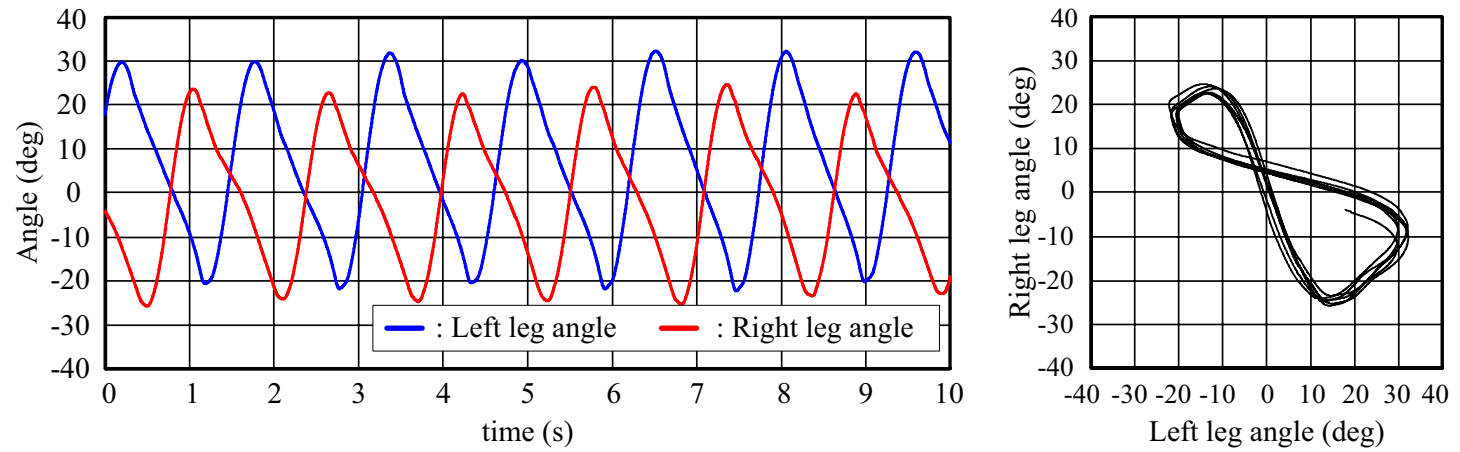

(a) Trajectories of leg angle in phase plane and timeline when the slope angle is 2.0 deg
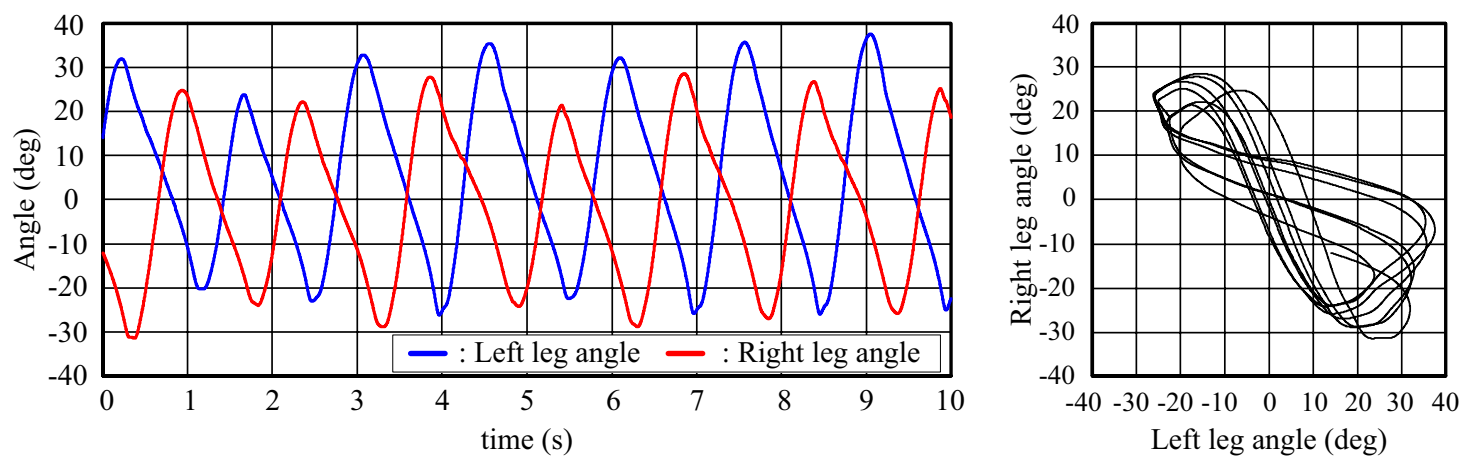

(b) Trajectories of leg angle in phase plane and timeline when the slope angle is $2.5 \mathrm{deg}$

Fig. 5 Experimental results of passive dynamic walking

was set to $0.0142 \mathrm{~N}-\mathrm{m} /(\mathrm{rad} / \mathrm{s})$ for the normal viscous-friction setting.

The experimental results are summarized in Fig. 6 in histogram format, and the average value and standard deviation of the stride angle were calculated. The histograms are normalized to 120 steps because the walking step count depends on the experimental condition. It can be confirmed that the stride angle increases as the walking slope angle increases, and the variation of the stride angle also increases, as shown in the histograms. In addition, not only the average value and the variation of the stride angle increase when the inclination angle of the walking slope was $3.0^{\circ}$, but also two peak stride angles seem to appear in the histogram, as shown in Fig. 6d. On the basis of these results, we summarize the relationship between the walking slope angles and the stride angles in Fig. 7.

The phenomenon that the stride angle increases as the walking slope inclination angle increases has been confirmed by the numerical simulation results of several conventional research studies [1-3, 8]. Similarly, in the experiment, it is also confirmed that the stride angle becomes unstable when the walking slope angle exceeds a certain value as shown in Fig. 7. In addition, it can be estimated that the stride angles bifurcate.

Therefore, it is considered that the results of this experiment are in good agreement with the results of conventional research studies.

\subsection{Relationship between the viscosity friction coefficient of the hip joint and the stride angle}

Here, we investigated the effect of the viscosity friction coefficient change of the hip joint by several experiments. The experiments were performed under the same conditions presented as in Sect. 4.1, except that the viscosity friction coefficient of the hip joint was set to $0.0242 \mathrm{~N}-\mathrm{m} /(\mathrm{rad} / \mathrm{s})$. Figure 8 shows the histogram plots of all results of the experiment. Note that these histograms tend to show a negative skew. When the robot walked with a large stride angle in this experiment, it showed unstable behavior and rarely finished walking the distance of $8 \mathrm{~m}$. On the other hand, the experimental data were collected when it finished walking $8 \mathrm{~m}$. Thus, the number of steps with a large stride angle decreased and the negative skew appeared in the histograms. 


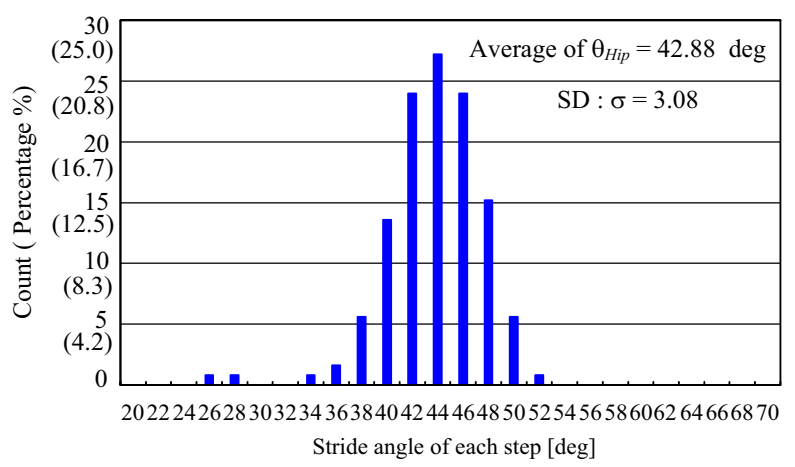

(a) slope angle $=2.0 \mathrm{deg}$, viscous friction : $0.0142 \mathrm{~N}-\mathrm{m} /(\mathrm{rad} / \mathrm{s})$

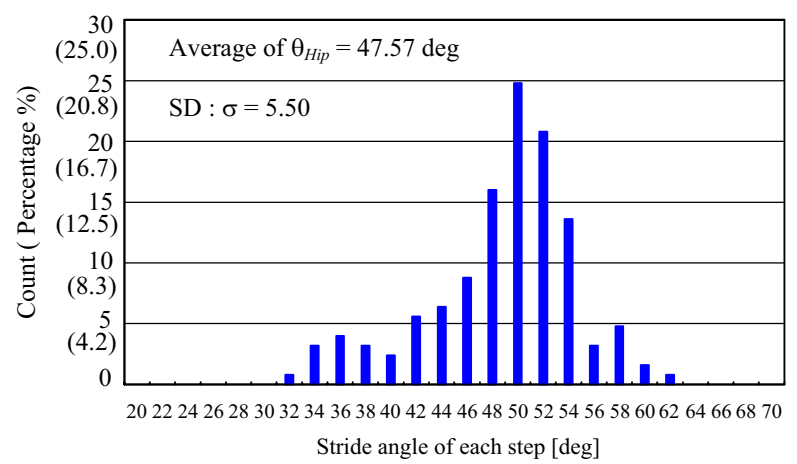

(b) slope angle $=2.5 \mathrm{deg}$, viscous friction : $0.0142 \mathrm{~N}-\mathrm{m} /(\mathrm{rad} / \mathrm{s})$

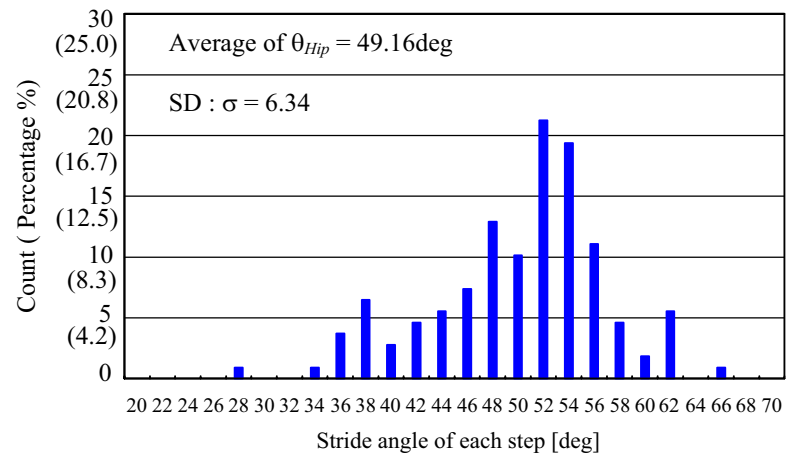

(c) slope angle $=2.7 \mathrm{deg}$, viscous friction : $0.0142 \mathrm{~N}-\mathrm{m} /(\mathrm{rad} / \mathrm{s})$

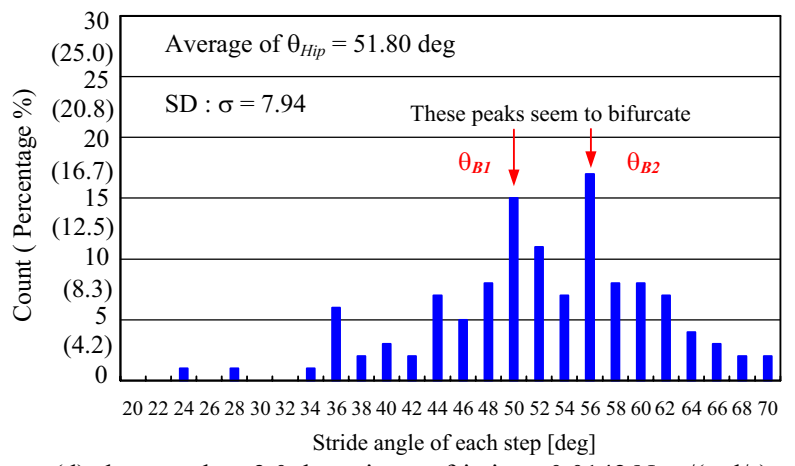

(d) slope angle $=3.0 \mathrm{deg}$, viscous friction : $0.0142 \mathrm{~N}-\mathrm{m} /(\mathrm{rad} / \mathrm{s})$

Fig. 6 Histograms of the stride angle in experiment with viscosity friction coefficient of $0.0142 \mathrm{~N}-\mathrm{m} /(\mathrm{rad} / \mathrm{s})$

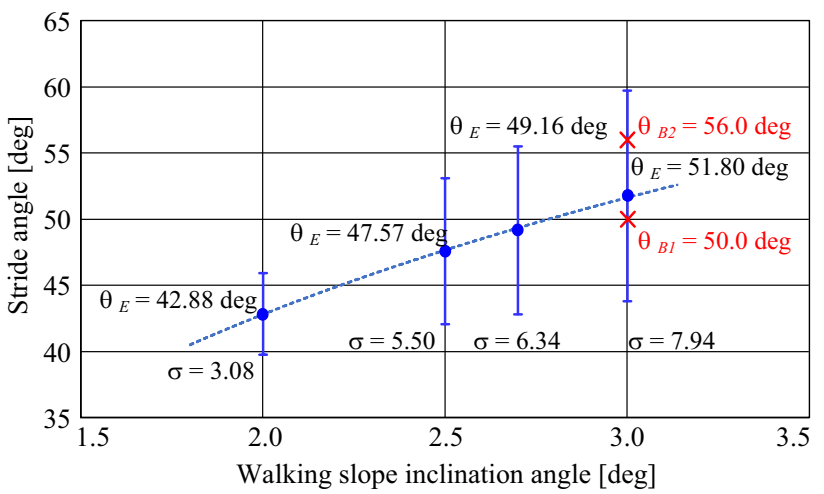

Fig. 7 Stride angle transition against walking slope inclination angle

It seems that the average value of the stride angle tends to decrease, as shown by comparing the results of Fig. 8 with those of Fig. 6. In addition, since the standard deviation also tends to decrease, much of the stride angle tends to concentrate around its average value when the viscosity friction coefficient of the hip joint increases. From these results, it can be seen that the viscous-friction increase of the hip joint has the effect of suppressing the stride angle and its variation during walking. The results also suggest that the viscous-friction increase of the hip joint not only reduces the stride angle and its variation but also improves the stability of walking motion, which was also observed in conventional research studies [9].

\subsection{Verification of adaptive behavior}

We verified the adaptive behavior by several experiments. Numerical simulations of previous research studies showed the existence of adaptive behavior; the robot changes its stride angle to continue the walking motion automatically by itself, when the inclination angle of the walking slope or the viscosity coefficient of the hip joint changes during walking [8-10].

Since the adaptive behavior appears with not only environmental changes but also changes in the internal parameters of the legged robot, we provided two experimental conditions for verification, as shown in Fig. 9. The left figure in Fig. 9 shows the experimental environment for investigating the adaptive behavior toward changes in the walking slope angle. The right figure in Fig. 9 shows the environment for investigating the adaptive behavior toward changes in the viscosity friction coefficient of the hip joint.

The first verification experiment was performed 10 times. The inclination angle of the walking slope was changed from $2.0^{\circ}$ to $2.5^{\circ}$ at the middle point $(4.0 \mathrm{~m}$ point on the walking slope) for the experiment. Figure 10a shows the result of the experiments; the 70 steps on the walking slope with a $2.0^{\circ}$ 


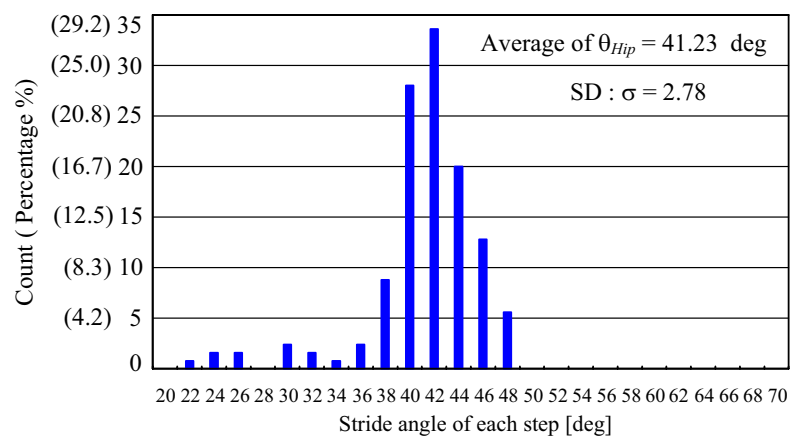

(a) slope angle $=2.0 \mathrm{deg}$, viscous friction : $0.0242 \mathrm{~N}-\mathrm{m} /(\mathrm{rad} / \mathrm{s})$

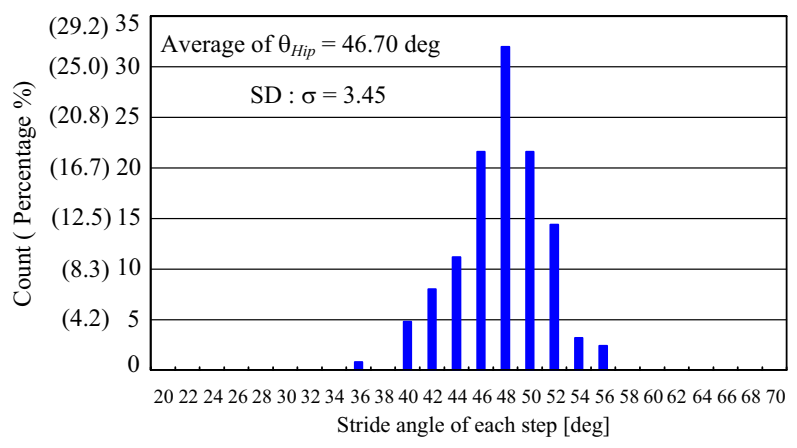

(b) slope angle $=2.5 \mathrm{deg}$, viscous friction : $0.0242 \mathrm{~N}-\mathrm{m} /(\mathrm{rad} / \mathrm{s})$

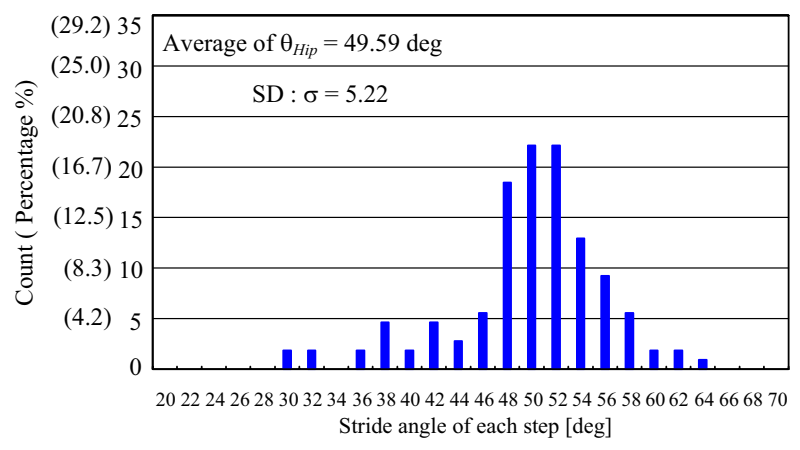

(c) slope angle $=2.7 \mathrm{deg}$, viscous friction : $0.0242 \mathrm{~N}-\mathrm{m} /(\mathrm{rad} / \mathrm{s})$

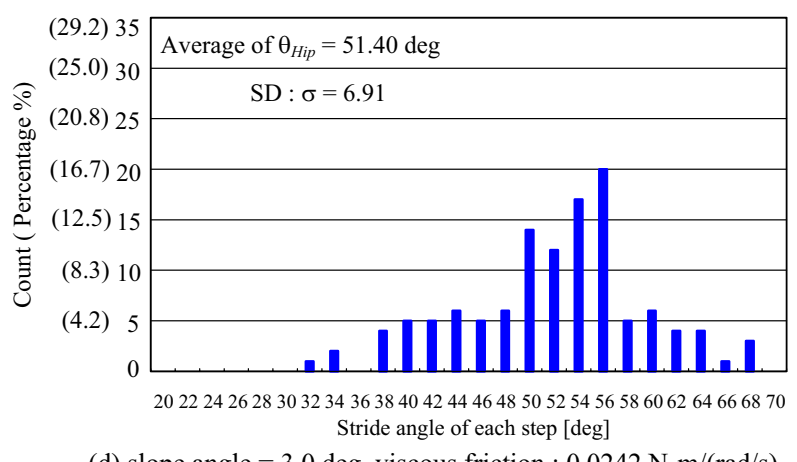

(d) slope angle $=3.0 \mathrm{deg}$, viscous friction : $0.0242 \mathrm{~N}-\mathrm{m} /(\mathrm{rad} / \mathrm{s}$ )

Fig. 8 Histograms of stride angle in experiment with viscous friction of $0.0242 \mathrm{~N}-\mathrm{m} /(\mathrm{rad} / \mathrm{s})$ inclination angle are in the first half, and the 60 steps on the walking slope with $2.5^{\circ}$ inclination angle are in the remaining half. In this experiment, the stride angle data for 150 steps were collected in total; however, the stride angle at the time of passing through the middle point showed momentary unstable behavior, so the two steps around the point were eliminated. This is because we confirmed that the stride angle became less variable while the robot was walking two steps during the transition to the next condition.

We compare the results shown in Fig. 10a with those in Fig. $6 \mathrm{a}, \mathrm{b}$. The average value of the stride angle in the first half was $42.26^{\circ}$ and that in the remaining half was $46.38^{\circ}$, as shown in Fig. 10a. The differences between the average values of the stride angles shown in Fig. 6a, $\mathrm{b}$ and the values shown in Fig. 10a are very close to an error of $-1.45 \%$ in the first half and close to an error of $-2.57 \%$ in the remaining half. From this experiment, the continuous transitions of the walking motion with changing stride angle at the middle point as a boundary are confirmed. In this experiment, average values of the stride angle in the first half and the remaining half are slightly smaller than the results in Fig. 6a, b. This is considered to be the range of error due to very subtle changes in experimental conditions. The result shows that the adaptive behavior actually appears in the experimental environment.

The second verification experiment was also performed 10 times. The viscosity friction coefficient of the hip joint was changed from 0.0142 to $0.0242 \mathrm{~N}-\mathrm{m} /(\mathrm{rad} / \mathrm{s})$ at the middle point ( $4.0 \mathrm{~m}$ point on the walking slope) during walking, as shown in the right figure of Fig. 9. The inclination angle of the walking slope was set to $2.0^{\circ}$ for this experiment. The same as in the previous experiment, the data of 70 steps were obtained in the first half and those of 60 steps were obtained in the remaining half. In this experiment, the stride angle data for 150 steps were collected in total. However, the stride angle data at the time of the viscosity friction coefficient change showed instantaneous unstable behavior, so the two steps around that timing were eliminated. The summary of the experiment is shown in Fig. 10b. From the result, it can be confirmed that the stride angle continuously transitions with the change in the viscosity friction coefficient of the hip joint during walking.

We compare the results shown in Fig. 10b with those in Figs. 6a, 8a. The average value of the stride angle in the first half is $42.31^{\circ}$ and that in the remaining half is $40.90^{\circ}$ in Fig. 10b. The difference between the average values of the stride angles in the first half shown in Fig. 10b and the values shown in Fig. 6a are very close within the error of $-1.34 \%$. The difference between the average values of the stride angles in the remaining half shown in Fig. 10b and the values shown in Fig. 8a are very close within the error of $-0.78 \%$. In this experiment, average values of the stride angle in both the first half and the remaining half are slightly 

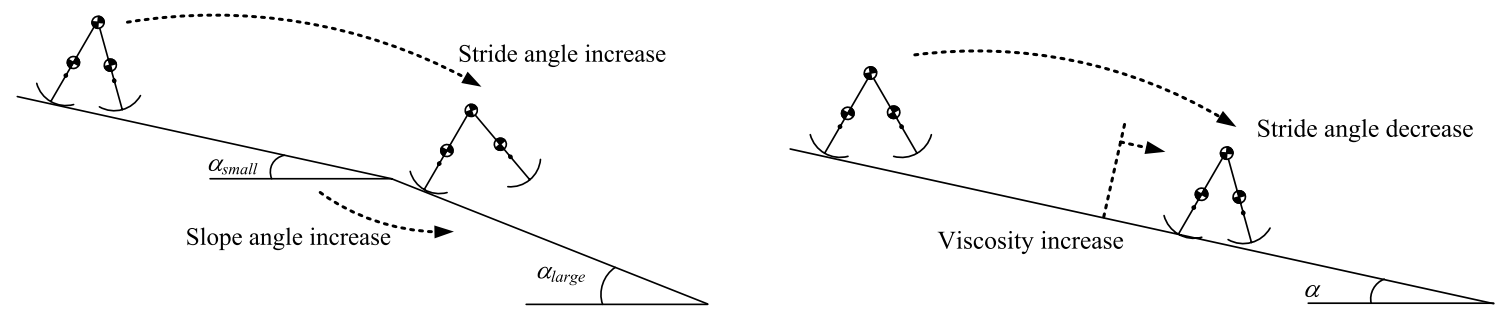

Fig. 9 The left figure shows the experimental environment for investigating the adaptive function for changing the inclination angle of the walking slope, and the right figure shows the environment for investigating the adaptive function for changing the viscosity of the hip angle
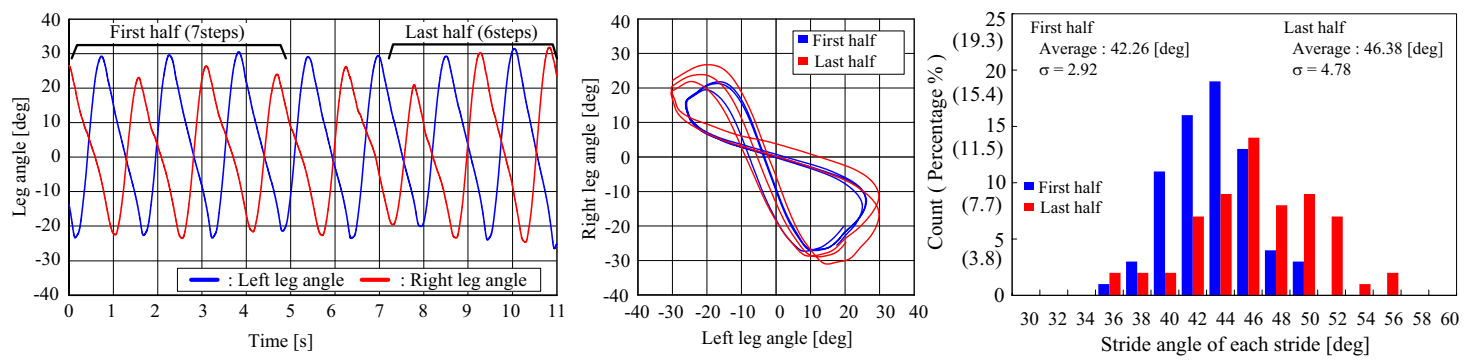

(a) Trajectories in timeline and phase plane, and histogram of stride angles when the inclination angle of the walking slope increases from 2.0 to $2.5 \mathrm{deg}$ during walking at viscosity friction coefficient of $0.0142 \mathrm{~N}-\mathrm{m} /(\mathrm{rad} / \mathrm{s})$
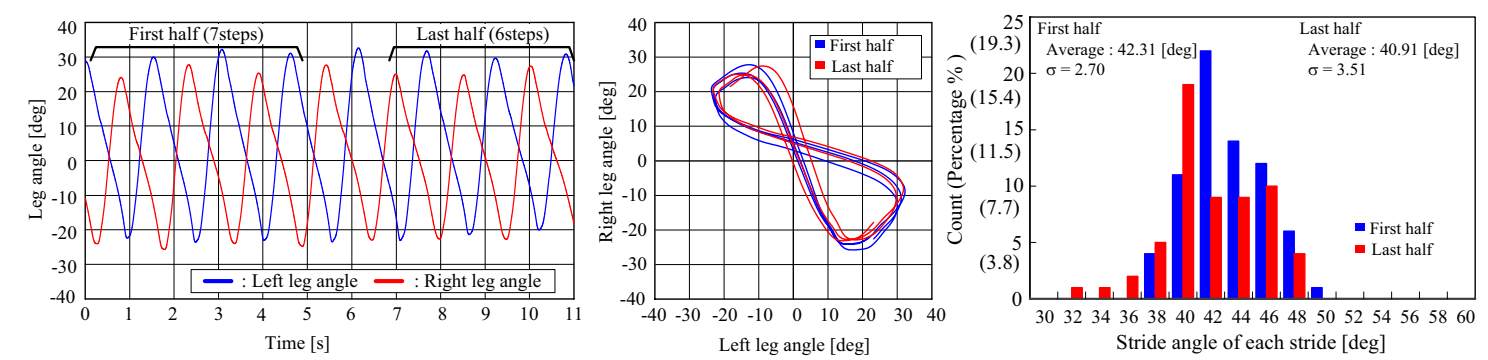

(b) Trajectories in timeline and phase plane, and histogram of stride angles at slope angle of 2.0 deg when viscosity friction coefficient increases from 0.0142 to $0.0242 \mathrm{~N}-\mathrm{m} /(\mathrm{rad} / \mathrm{s})$ during walking

Fig. 10 Experimental results of investigating the adaptive behaviors of passive dynamic walking

smaller than the results in Figs. 6a, 8a. This is also considered to be the range of error due to very subtle changes in experimental conditions, same as the above experiment.

From the experiment, the same as above, the continuous transitions of the walking motion with the change in stride angles at the middle point as a boundary are confirmed. These results suggest that the adaptive behavior, which is caused by the change in the internal parameters of the legged robot, appears actually in the experimental environment. In addition, referring the numerical simulations in conventional studies, transitions among the simulative conditions caused by the adaptive behavior are reversible $[5,6]$. Thus, even in the experimental conditions, it can be expected that the transitions of the adaptive behavior are also reversible.

\section{Conclusions}

In this study, we developed a legged robot and a walking slope for use in experiments on passive dynamic walking, and the same behaviors that were observed by numerical simulations in conventional research studies were actually reproduced by using these devices. Results indicate that the characteristic behaviors of passive dynamic walking, which were shown in the conventional research studies by simulation, actually exist.

Although many studies on passive dynamic walking are conducted by numerical simulations, it is considered to be a major achievement to show that the results of conventional studies also exist in the real world. 
Particularly for adaptive behavior, we showed that the size and degrees of freedom of a legged robot could be designed by applying the adaptive behavior in computer simulations. It is considered that the validity of this design method can be confirmed by the results of experiments.

In addition, we also confirmed that the stability of walking motion was improved by adjusting the viscosity friction coefficient of the hip joint obtained in a previous study by numerical simulations. The results of this study indicate that it is effective to adjust the viscosity friction coefficient of the joint as a method of stabilizing walking motion in the real world. For future work, we will apply the knowledge obtained from these experiments to the design theory of legged robots.

Open Access This article is licensed under a Creative Commons Attribution 4.0 International License, which permits use, sharing, adaptation, distribution and reproduction in any medium or format, as long as you give appropriate credit to the original author(s) and the source, provide a link to the Creative Commons licence, and indicate if changes were made. The images or other third party material in this article are included in the article's Creative Commons licence, unless indicated otherwise in a credit line to the material. If material is not included in the article's Creative Commons licence and your intended use is not permitted by statutory regulation or exceeds the permitted use, you will need to obtain permission directly from the copyright holder. To view a copy of this licence, visit http://creativecommons.org/licenses/by/4.0/.

\section{References}

1. McGeer T (1990) Passive dynamic walking. IJRR 9(2):62-82

2. Goswami A, Thuilot B, Espiau B (1996) Compass-Like Biped Robot-Part I: Stability and Bifurcation of Passive Gaits. Technical Report 2996, INRIA
3. Garcia M, Chatterjee A, Ruina A, Coleman M (1998) The simplest walking model: stability, complexity, and scaling. J Biomech Eng 120(2):281-288

4. Osuka K, Kirihara K (2000) Motion analysis and experiment of passive walking robot quartet II. J Rob Soc Jpn 18(5):737-742 (In Japanese)

5. Hirata K (2011) On internal stabilizing mechanism of passive dynamic walking. SICE J Control Meas Syst Integr 4(1):29-36

6. Hayashi T, Kaneko K, Asano F, Luo ZW (2011) Experimental study of dynamic bipedal walking based on the principle of parametric excitation with counterweights. Adv Robot 25(1-2):273-287

7. Owaki D, Koyama M, Yamaguchi S, Kubo S, Ishiguro A (2011) A 2-D passive-dynamic-running biped with elastic elements. IEEE Trans Rob 27(1):156-162

8. Iribe M, Osuka K (2006) A designing method of the passive dynamic walking robot via analogy with the phase locked loop circuits. In: Proc. of ROBIO2006. Kunming, pp 636-641

9. Iribe M, Osuka K (2007) Design of the passive dynamic walking robot by applying its dynamic properties. J Rob Mechatron 19(4):402-408

10. Ura D, Iribe M, Osuka K, Kinugasa T (2015) Legged walking robot design applying a behavior of passive dynamic walkingjoint D.O.F alignment design applying the adaptive function. Trans SICE 51(5):329-335 (In Japanese)

11. Ura D, Sugimoto Y, Sueoka Y, Osuka K (2017) Asymptotic realization of desired control performance by body adaptation of passive dynamic walker. J Rob Mechatron 29(3):480-489

12. Iribe M, Furukawa H, Sugimoto Y, Kinugasa T, Osuka K (2011) Prototype passive walker development which has capabilities to measure the state parameters of its own. In: Proceedings of the 14th international conference on climbing and walking robots and the support technologies for mobile machines (CLAWAR2011). Paris, France, pp 581-588

Publisher's Note Springer Nature remains neutral with regard to jurisdictional claims in published maps and institutional affiliations. 